\title{
CAMBIOS EN LAS FORMAS DEL COMERCIO Y EL CONSUMO EN BARCELONA
}

\author{
POR \\ CARLES CARRERAS I VERDAGUER \\ JORGE ROMERO GIL
}

Este artículo presenta los cambios en las formas de comercio y consumo en la ciudad de Barcelona a lo largo del siglo xx, a partir del papel que han tenido las diversas innovaciones económicas y técnicas y sus consecuencias sociales. Se presenta un análisis histórico de la evolución del equipamiento comercial barcelonés, dentro del marco español y europeo con el fin de destacar las formas de adaptación local de los procesos de globalización que están en marcha. El estudio se basa en una dilatada experiencia de investigación en este campo que cuenta ya con numerosas publicaciones.

\section{Comercio y consumo en Barcelona, unos precedentes}

A principios del siglo $\mathrm{xx}$, aparece en Barcelona la que fuera una de las grandes innovaciones de la actividad comercial contemporánea: los primeros grandes almacenes. Se localizaron en la Plaza de Catalunya y sus alrededores (Avda. del Portal de l'Àngel, las Ramblas, calle Pelai), hoy en pleno corazón de la ciudad y que era el ámbito que representó el desplazamiento del centro comercial de la ciudad medieval a la nueva, hacia el

\footnotetext{
Carles Carreras i Verdaguer: Catedrático de Geografía Humana. Universitat de Barcelona.

Jorge Romero Gil: Becario de Geografía Humana. Universitat de Barcelona.

Estudios Geográficos

Tomo LXI, 2000, n. ${ }^{\circ} 238$, enero-marzo 
ensanche Cerdà del siglo XIx. En primer lugar, fueron los almacenes de Vicenç Ferrer, situados en parte en el espacio que ocupa actualmente El Corte Inglés; después fue la empresa manresana Jorba, que inauguró en 1926 su sede del portal de l'Àngel, edificio que puede considerarse, desde el punto de vista arquitectónico, como los grandes almacenes más representativos de la ciudad; en las Ramblas se situaron los almacenes Sepu, los únicos que continúan en la actualidad; en la calle Pelai los almacenes el Siglo, hoy C\&A, los Almacenes Alemanes, después Capitolio y hoy desaparecido y El Águila, destruidos por un incendio en 1985 y hoy edificio residencial.

Estos grandes almacenes supusieron una primera gran transformación en el sector del comercio al por menor de la ciudad. Construidos en la arquitectura del hierro y del vidrio, especialmente, Jorba, el Siglo o El Águila, siguiendo tarde y lejanamente la moda del Crystal Palace de Londres, que permite levantar grandes edificios con vacíos interiores amplios y combinar la iluminación natural y artificial, significaron mucho más que la posibilidad industrial de venta a gran escala. Como es habitual, el cambio de escala significa también la introducción de cambios estructurales importantes en otros aspectos, sobre todo en las técnicas de venta. Los grandes almacenes organizan la oferta de sus mercancías muy diversificadas a partir del acceso directo del público, sin el intermediario vendedor (dueño o empleado), ni el mostrador tradicional; los consumidores pueden pasear así entre los objetos del deseo, compran o no compran, observan y tocan, se informan y prueban, y acaban a veces comprando. El gran almacén, así, supone la incorporación de las técnicas y características de la empresa privada y de un espacio cerrado al carácter cultural y de ocio de la visita a los mercados tradicionales y de los espacios públicos, en general, innovación que no ha hecho sino profundizarse con el tiempo.

Estas innovaciones tuvieron como eje la ciudad de Barcelona; el comportamiento del incipiente espacio metropolitano, en esta fase, era tan sólo el de un espectador de lo que acontece en la capital. En ese sentido, algunos lugares eran especialmente visitados por los habitantes de los municipios cercanos a Barcelona, como es el caso, por ejemplo, de la calle Pelai o, en otro contexto - pues la actividad comercial desarrollada allí es diferente de la del centro de la ciudad - la calle de Sants, fronteriza con la localidad de l'Hospitalet de Llobregat. Este factor de atracción del centro comercial se resume muy bien en una frase, utilizada no sólo por 
los no barceloneses sino también por muchos de los habitantes de los barrios más o menos periféricos de la misma ciudad, se trata de la expresión «ir de compras a Barcelona» 0 «bajar a Barcelona ${ }^{1} »$. En este concepto se resume el carácter lúdico, pues se incluye la idea de la visita, de paseo y la compra más o menos extraordinaria, y de acontecimiento excepcional que ese «bajar a Barcelona» conlleva.

La evolución del consumo barcelonés ha de contar, al menos, con dos premisas fundamentales. Una, la existencia de una oferta comercial muy diversificada basada en una estructura económica muy fragmentada en multitud de pequeñas y medianas empresas de carácter familiar; es lo que da la base a una Barcelona ciudad de tenderos, sociológicamente muy importante $^{2}$. La otra, es una de las manifestaciones en la ciudad de las formas del estado del bienestar, institucionalizada con continuidad en la administración local, ante la falta de participación de estructuras administrativas de rango superior y de la empresa privada: la red de mercados municipales; unas decenas de mercados bien distribuidos abastecen el consumo cotidiano y constituyen auténticos centros sociales en la mayoría de los barrios de la ciudad.

El mismo modelo de la capital se repite en su área metropolitana, municipio a municipio; ahí se presenta también una estructura comercial de empresas familiares, casi de empleo hereditario, de pequeños comercios que son «las tiendas del barrio» cuya oferta queda complementada, sobre todo en lo que se refiere al sector alimentación, por uno o varios mercados municipales, dependiendo de la entidad de la población. No obstante, esta homogeneidad de estructuras comerciales entre la ciudad de Barcelona y las poblaciones de su área metropolitana no reflejan necesariamente una interacción entre la ciudad y su corona, sino tan sólo un cierto mimetismo, ya que hasta los años 1960 no se completaría la simbiosis funcional entre Barcelona y las poblaciones que la rodean; la homogeneidad en las formas del comercio y el consumo no son más que el reflejo de una homogeneidad social catalana más general.

${ }^{1}$ Radio Barcelona emitió durante muchos años un programa matinal todos los domingos con el expresivo título de «La comarca nos visita», patrocinado por algunas empresas del sector alimentario, como los caramelos Fradera de Mataró, o los enlatados Tres Pins.

${ }^{2}$ Esta Barcelona de tenderos fue magníficamente caricaturizada por Santiago Rusiñol en su conocida obra L'Auca del Senyor Esteve, de 1907. 
Las estructuras comerciales reflejan así una sociedad ya plenamente urbana pero con unos fuertes vínculos tradicionales, donde cada localidad y cada barrio forman su propio mundo cotidiano, con «sus» establecimientos, «sus» comerciantes y «sus» consumidores. Estos elementos lejos de ser anónimos son perfectamente identificables por sus nombres e incluso sus alias, estableciéndose unas relaciones de proximidad entre todos los agentes que de alguna manera condicionan la relación comercial, que se convierten también en una relación social importante. En esta realidad se reflejan aún unos usos del tiempo y del espacio muy definidos, cuya relación se encuentra claramente delimitada; así, el tiempo cotidiano, el tiempo de la compra diaria, se resumía y se consumía en el espacio más cercano, el del propio barrio o localidad, en un contexto en el que la mayoría de la población no disponía de medio propio de transporte, ya que hasta bien entrados los años sesenta el automóvil fue un lujo extraordinario; el tiempo de la compra cotidiana era también el tiempo que se consumía en los establecimientos tradicionales locales. Igualmente, la posibilidad de la compra semanal de los productos básicos, esencialmente alimenticios, no era ni necesaria ni funcional, en la medida de la escasa incorporación de la mujer al mundo laboral por lo que ella era la encargada de la compra diaria, como parte de lo que eufemísticamente se denominaba sus labores, así como de la no disposición de medio de transporte propio en el que, además de desplazarse, pudiera colocarse la carga de la teórica compra semanal. En la práctica existían pues sólo dos tipos de comportamientos de consumo: la compra cotidiana en tiendas locales tradicionales o en los mercados municipales, y la compra excepcional que se realizaba esencialmente en el centro de Barcelona, tanto para los habitantes del área metropolitana como para los de la propia ciudad ${ }^{3}$, en un tiempo más dedicado al ocio que a la cobertura de necesidades.

Es este mundo el que se vio afectado por los cambios sociales y económicos, que empezaron a generarse hacia 1950, a partir del plan de estabilización a escala estatal y de la instalación de la factoría automovi-

${ }^{3}$ Esta actitud de considerar el centro de la ciudad como el lugar donde se realizan las «compras extraordinarias» con una periodicidad relativamente larga, como puede ser la mensual, se daba incluso entre los habitantes de barrios cercanos al centro urbano, como, por ejemplo, es el caso de los habitantes del barrio de Santa Catalina que declaraban taxativamente «tenemos en este barrio lo que muchos no tienen. y no tenemos que desplazarnos porque lo tenemos dentro» (López, P., 1986). 
lística SEAT ${ }^{4}$ a escala barcelonesa. Los cambios se incrementaron con el llamado desarrollismo de la década de los sesenta, en la que se generaliza el modelo fordista en España, con todas sus consecuencias, de auténtica revolución burguesa.

\section{La revolución burguesa de los años sesenta}

El desarrollismo de los años 1960 ha marcado una frontera clara en la historia económica, política y cultural del consumo para Barcelona y para el conjunto de España. Gracias a la llegada del turismo, además, se pasó a contar, de forma relativamente indiscriminada, con una escuela práctica y cotidiana de modernización a través de nuevos objetos y nuevos hábitos cotidianos. También se dieron unos primeros cambios, si bien en principio tímidos y lentos, en las infraestructuras comerciales y en las formas de consumo, al menos en Barcelona y en su entorno metropolitano; cambios que alteraron el comercio local. Estudiar estos cambios resulta hacer una historia sobre la que prácticamente no existe documentación de ningún tipo; como geógrafos sólo queda el recurso de analizar los restos espaciales de los cambios, cosa que permite una cierta aproximación sincrónica, y poco dinámica tal vez, a la realidad territorial, formada de fragmentos social y temporalmente diferentes (Carreras, 1993; 1995).

En los años 1960, la modernización comercial se fue introduciendo a través de formas de cooperación entre las pequeñas empresas, así como de algunos intentos limitados de participación en la ordenación del espacio urbano por parte de algunos empresarios con más iniciativa. En esta época se asiste a una expansión del centro comercial que, desde la plaza de Cataluña, se extiende a lo largo del paseo de Gracia primero, y de la avenida Diagonal después, hasta alcanzar la plaza de Francesc Macià ${ }^{5}$ y, posteriormente, incluso Maria Cristina., alcanzando los emblemáticos 5 $\mathrm{km}$. de longitud. Esta expansión fue protagonizada por el crecimiento de actividades comerciales diversas y por la ubicación de sedes financieras, que en ocasiones desalojaron antiguas tiendas de lujo. Desde entonces la

\footnotetext{
${ }^{4}$ Que, entre otras muchas consecuencias sociales y económicas, implica la generalización del uso del automóvil.

${ }^{5}$ Denominada entonces significativamente de Calvo Sotelo.
} 
asociación de la actividad financiera y el comercio ha sido una constante bajo diferentes formas, desde el cambio de actividad de algunos edificios hasta la implicación del capital financiero en determinados proyectos comerciales. En los inicios de los sesenta irrumpen en el panorama barcelonés las principales cadenas estatales de grandes almacenes, en 1962 El Corte Inglés se instaló en la plaza Cataluña, y en 1964 lo hizo Galerías Preciados, que absorbió a los antiguos almacenes Jorba. Esta expansión de los grandes almacenes fue acompañando a la del centro comercial; así en 1963, la empresa americana Sears se instaló en la Diagonal, junto a la plaza Macià, y, en 1974, El Corte Inglés ${ }^{6}$ hizo lo propio en la plaza María Cristina, junto a la sede de la principal entidad financiera catalana, La Caixa. Estas cadenas de grandes almacenes no sólo amplían la oferta cuantitativamente sino que modifican también las formas de compra, al introducir procesos de capitalización a gran escala, los pagos aplazados y las tarjetas de crédito.

Junto al crecimiento de los grandes almacenes, las galerías comerciales, como agrupación de pequeñas tiendas, fueron la fórmula de éxito que acompañó a los cambios técnicos del autoservicio, introducidos en los pequeños establecimientos de alimentación. Al mismo tiempo, l'illa de vianants, una zona del centro de Barcelona donde se restringió el tráfico rodado y se fomentó la idea de «pasear e ir de compras», constituyó la primera operación de urbanismo concertado a cargo sobre todo de la iniciativa privada local ${ }^{7}$. En los años 1970, con posterioridad a la cohesión industrial del espacio metropolitano barcelonés, se produjeron los primeros pasos en la integración de los espacios comerciales del área metropolitana y de Barcelona ciudad; en cierta medida, la señal de salida la marcó, en 1971, el traslado del mercado central de abastos de Barcelona desde el Borne, en el casco antiguo, hasta Mercabarna -en la periférica Zona Franca, abierta hacia el Baix Llobregat, una de las áreas más industriales de la corona metropolitana. Entre finales de los años setenta e inicios de los ochenta se implantaron las dos primeras grandes superficies de la corona metropolitana,

\footnotetext{
${ }^{6}$ Esta empresa ha acabado por ser la dominante en el panorama español de grandes almacenes, en el ámbito estatal absorbió en 1995 a Galerías Preciados, que a su vez había absorbido a los establecimientos que Sears tenía en España (Cuartas, J., 1992).

${ }^{7}$ Llama la atención la falta de reflexión sobre l'illa de vianants (isla peatonal) de Barcelona en cualquier estudio urbanístico de la ciudad y, más aún, del distrito de Ciutat Vella, como mínimo en los publicados; este hecho es todavía más significativo cuando la colaboración entre autoridades municipales y empresarios comerciales hace tiempo que da frutos de urbanismo concertado y estratégico.
} 
el hipermercado Hyper ${ }^{8}$, situado en el Prat de Llobregat en la primera corona, y Baricentro ${ }^{9}$, situado en Barberà del Vallès, en la segunda corona, que podría ser considerado como el primer shopping center que funcionó en España. En este mismo período se generalizó masivamente, el uso de dos bienes de consumo prácticamente infraestructurales en la vida moderna: el automóvil y la nevera; este hecho implicó dos factores esenciales de cambio en el consumo: el aumento espectacular de la movilidad de los consumidores y el de la conservación durante más tiempo de los alimentos adquiridos lo que permite la posibilidad de la compra semanal o mensual.

\section{Los cambios a partir de 1986}

La iniciativa pública, esencialmente municipal, ha liderado el desarrollo de los proyectos olímpicos y de los dos planes estratégicos de Barcelona, a partir de mediados de los años 1980. Estos se han centrado primordialmente en la construcción de nuevos espacios públicos de los que la ciudad era claramente deficitaria. Por una parte, se quería suturar las cicatrices que el crecimiento sin demasiado planeamiento, y con menos control dernocrático, de los años sesenta había originado, mientras que, por otra parte, se trataba de absorber los solares y locales que la desindustrialización dejaba vacíos ${ }^{10}$. Barcelona era una ciudad muy densa, con pocos espacios abiertos; así, plazas públicas, grandes espacios, parques y jardines han sido incorporados a la ciudad, junto a un nuevo mobiliario urbano y monumentos artísticos que se han distribuido por toda la ciudad ${ }^{11}$. La monumentalización se ha centrado especialmente en

${ }^{8}$ Este centro se mantiene en la actualidad, incluso se ha expandido, pero ha pasado por diferentes manos, de Hyper paso a Carrefour y, finalmente, a la cadena francesa Pryca, con la que se denomina Pryca Prat.

${ }_{9}$ Este centro fue realizado en dos fases, la primera se inauguro en abril de 1980 y constaba de $46.000 \mathrm{~m}^{2}$ de superficie comercial, la segunda se completó en abril de 1982 y constaba de $26.800 \mathrm{~m}^{2}$ de superficie comercial, dando un total de $72.800 \mathrm{~m}^{2}$ de superficie comercial; el centro cuenta con un total de 150 locales y 4.000 plazas de aparcamiento.

${ }_{10}$ Hoy numerosos parques y jardines y algunos equipamientos culturales de Barcelona y de las ciudades de su alrededor conservan todavía el nombre de las viejas industrias ochocentistas, como los parques de La España Industrial o Pegaso de Barcelona o La Farga o Tecla Sala, de l'Hospitalet de Llobregat.

${ }_{11}$ De la importancia de estas acciones da fe el hecho que los espacios públicos de Barcelona ganasen el premio príncipe de Gales 1990, concedido por la facultad de arquitectura de la Universidad de Harvard y el de la real asociación de arquitectos británicos en 1999. 
el ámbito de los espacios culturales, parques y jardines, museos y bibliotecas, teatros y auditorios que dotan a la ciudad de nuevas formas, multiplican y afirman la oferta turística de una ciudad ya integrada en los mercados internacionales y complementan su oferta comercial. Esta política ha permitido la plasmación de un planeamiento que formalmente podría ser considerado el propio de la postmodernidad, produciendo una identificación muy fácil y discutible entre ésta y la ciudad postfordista (Jameson).

Durante los años 1990 aparecen con fuerza los grandes espacios comerciales, coincidiendo con la aprobación de una serie de nuevos planes estratégicos en numerosos municipios del área metropolitana (Terrassa en 1993, Viladecans en 1994, Sant Boi de Llobregat en 1995) mientras se hace balance y revisión del plan estratégico de Barcelona. En estos planes estratégicos, respondiendo a una concepción de ciudad-empresa, el comercio y el sector comercial resultan elementos esenciales dentro de los procesos que configuran la nueva ciudad terciaria siguiendo el modelo denominado postfordista. La coyuntura de cambios internacionales generada por la mundialización y flexibilización de la economía explica la aparición de estas nuevas dotaciones comerciales, y lo hace en un momento en que ya ha entrado en crisis el uso de los espacios públicos urbanos tradicionales, a favor de espacios privados, cerrados y seguros, artificialmente climatizados y discretamente vigilados. Así la distinción conceptual entre privado y público se difumina, mientras que las iniciativas y capitales de ambos sectores colaboran en la mayor parte de los proyectos en marcha, construyendo unos nuevos escenarios para el consumo en la ciudad (Carreras, 1995).

En la ciudad de Barcelona, primero fue el moll de la Fusta donde la colaboración nada fácil entre el ayuntamiento y el puerto autónomo, que dependía de la administración periférica del Estado en aquellos momentos, permitió en 1990 la apertura a los ciudadanos como espacio de ocio de uno de los muelles más interiores del viejo puerto barcelonés. Este sector ha sido reforzado posteriormente, por iniciativa del puerto, con la remodelación completa del Port Vell, iniciada con la Marina en 1993, continuada en 1994 con la rambla de Mar, estructura móvil de madera que conecta el nuevo sector lúdico del puerto con la ciudad y el Imax, y ampliada en 1995 con el acuario y el Maremagnum, centro lúdico y comercial. En 1999, se finalizó la parte más exterior de estas nuevas instalaciones dedicada a un World Trade Center. 
El mismo proceso de apertura al público para actividades de ocio se ha desarrollado en la mayor parte de instalaciones olímpicas, con la excepción tal vez del estadio; clubes deportivos de barrio, bares y restaurantes, o actividades culturales esporádicas intentan sacar rendimiento de estos equipamientos, que, por otra parte, tienen un gran papel monumental, y una atracción popular para los turistas extranjeros y forasteros. El puerto olímpico, posiblemente por la proximidad del nuevo barrio formado en la antigua villa olímpica, ha sido el que ha disfrutado hasta ahora de una mayor utilización, como nueva área de ocio nocturno, especialmente en verano.

La iniciativa privada, incluso la internacional ${ }^{12}$, se sumó a esta transformación de los espacios urbanos, con la instalación de equipamientos exclusivamente dedicados a actividades comerciales a gran escala. Es el caso de la compañía suiza de seguros Winterthur, que en 1993 abrió en la Diagonal el centro comercial $L^{\prime} I l l a^{13}$, con los grandes almacenes Marks \& Spencer y el hipermercado Caprabo como anclas. El mismo año, los grandes almacenes japoneses Sogo se instalaban en el puerto olímpico, si bien esta experiencia se ha saldó con el cierre en 1996, siendo substituidos en 1999 por el casino de Barcelona. En 1994, el hipermercado vasco Eroski abrió un gran centro comercial ${ }^{14}$ en Cornellà de Llobregat, mientras que en 1995, Caprabo lo hacia en Gavà ${ }^{15}$ y fueron abiertos dos nuevos grandes centros comerciales en la ciudad de Barcelona: Glòries ${ }^{16}$, en los

12 De hecho, durante 1995 el sector de centros comerciales fue casi el único sector inmobiliario que despertó el interés de los inversores extranjeros (Richard Ellis, 1995).

${ }_{13}$ Las características del centro incluyen $135.000 \mathrm{~m}^{2}$ de superficie construida, 35.000 $\mathrm{m}^{2}$ de superficie comercial, $80.000 \mathrm{~m}^{2}$ de superficie de aparcamiento cubierta, 125 locales comerciales, 2.400 plazas de aparcamiento. Componen el complejo un centro comercial, que incluye centro de alimentación, grandes almacenes, hipermercado, galería comercial, equipamiento del hogar y equipamiento de la persona, un hotel, oficinas, un centro escolar, un centro de convenciones, locales de restauración y una discoteca y espacios lúdicos.

${ }^{14}$ Se trata del Llobregat Centre, que consta de $91.801 \mathrm{~m}^{2}$ de superficie construida, 28.490 $\mathrm{m}^{2}$ de superficie comercial y $47.244 \mathrm{~m}^{2}$ de superficie de aparcamiento, con 1671 plazas. Se compone de un centro comercial con hipermercado, galería comercial y tiendas especializadas, locales de restauración y multicines.

15 Es el Centro Comercial Barnasud, tiene $59.420 \mathrm{~m}^{2}$ de superficie construida, $28.700 \mathrm{~m} 2$ de superficie comercial y $54.691 \mathrm{~m}^{2}$ de superficie de aparcamiento, con 2.134 plazas. Componen el complejo un centro comercial, con hipermercado, galería comercial y superficies especializadas, locales de restauración, multicines y diferentes complejos lúdicos.

${ }^{16} \mathrm{Su}$ nombre es el Centre Comercial Diagonal/Glóries, tiene $47.457 \mathrm{~m}^{2}$ de superficie construida, $33529 \mathrm{~m}^{2}$ de superficie comercial y $126.112 \mathrm{~m}^{2}$ de superficie de aparcamiento con 3.686 plazas. Componen el complejo un centro comercial, con hipermercado, galería comercial y superficies especializadas, oficinas, zonas de restauración y multicines. 
antiguos locales de la fábrica Hispano Olivetti, y el ya citado Maremag$n u m^{17}$ en el Port vell. El año siguiente fueron otras localidades del área metropolitana, l'Hospitalet de Llobregat y Sabadell ${ }^{18}$, las que entraban en esta vía, mientras se discutía el caso de Sant Boi, al que se dió finalmente autorización para la apertura de un centro comercial. Paralelamente, los grandes almacenes de $E l$ Corte Inglés (que también se habían instalado en la zona del eje Macià en Sabadell) ampliaban su más antigua sede en Barcelona hasta ocupar toda una manzana de la plaza de Cataluña y la financiera francesa $U A P$ comenzaba la polémica construcción, acabada en 1998 de otra manzana de la plaza, entre las calles Pelai y Bergara, mientras que la firma Marks \& Spencer solicitaba instalarse en el viejo edificio del Banco Central, donde inauguró su establecimiento en 1999. Todo ello ha servido para reforzar la centralidad de la plaza y, como consecuencia, la vitalidad del denominado eje de los cinco kilómetros. En el mismo sentido, si bien dentro de una operación de alcance general, ha actuado la adquisición en 1995, por parte de El Corte Inglés, de los establecimientos que habían pertenecido a la cadena Galerías Preciados, su tradicional competidora en el sector español de grandes almacenes, situados en el Portal de l'Àngel y en la Plaza Francesc Macià de Barcelona.

Otras iniciativas se materializaron en 1996, como el centro comercial Centre de la Vil.la, en la antigua Villa Olímpica, o se encuentran en estudio en alguna de las denominadas áreas de nueva centralidad, como la zona de Diagonal-Mar o RENFE-Meridiana, mientras la remodelación de nuevos espacios, como los campos de fútbol de Sarrià y Nou, abren expectativas diferentes en el mismo sentido.

${ }_{17}$ Sus características generales son $29.255 \mathrm{~m}^{2}$ de superficie construida, $10.000 \mathrm{~m}^{2}$ de superficie comercial y $20.332 \mathrm{~m}^{2}$ de superficie de aparcamiento subterráneo, con 864 plazas. Componen el complejo un centro comercial, con $8.800 \mathrm{~m}^{2}$ para galería comercial, locales de restauración, bares, multicines, un cine Imax, espacio lúdico, oficinas y otras actividades.

${ }^{18}$ Se trata de tres nuevos centros comerciales. Uno, el Max Center, en el complejo de La Farga, en Hospitalet de Llobregat; este complejo cuenta con una superficie edificada de $44.000 \mathrm{~m}^{2}$, una superficie de venta de $12.793 \mathrm{~m}^{2}$, una superficie de aparcamiento de 24.000 $\mathrm{m}^{2}$, con un total de 960 plazas; consta de centro comercial, locales de restauración, un centro de convenciones y multicines. Otro en Sabadell, las galerías Paddock Bulevard, con 8.756 $\mathrm{m}^{2}$ de superficie edificada, $5.619 \mathrm{~m}^{2}$ de superficie de venta, 82 locales y $24.000 \mathrm{~m}^{2}$ de superficie de aparcamiento con 850 plazas; el complejo consta de un edificio de oficinas, una galería comercial y locales de restauración. Finalmente, el centro comercial Del Llac Center, con $10.650 \mathrm{~m}^{2}$ de superficie comercial, 102 locales comerciales, $9.028 \mathrm{~m}^{2}$ de superficie de oficinas y $9.422 \mathrm{~m}^{2}$ de superficie de aparcamiento, con 292 plazas; se compone de galería comercial, locales de restauración y ocio y edificio de oficinas. 
HIPERMERCADOS, GRANDES ALMACENES Y CENTROS COMERCIALES DE BARCELONA Y SU REGIÓN METROPOLITANA (1996)

HIPERMERCADOS mayores de $2.500 \mathrm{~m} 2$ de superficie (no ligados a centro comercial)

\begin{tabular}{|c|c|c|c|}
\hline Municipio & Empresa & $\begin{array}{l}\text { Superficie } \\
\text { venta }\left(\mathrm{m}^{2}\right)\end{array}$ & $\begin{array}{c}\text { Año } \\
\text { inauguración }\end{array}$ \\
\hline Manresa & Pryca & 9.487 & 1991 \\
\hline Cabrera de Mar & Pryca & 12.277 & 1992 \\
\hline El Prat de Llobregat & Pryca & 10.848 & 1973 \\
\hline Terrassa & Pryca & 9.463 & 1978 \\
\hline Sant Adrià del Besos & Pryca & 9.463 & 1987 \\
\hline Sant Cugat del Vallès & Pryca & 13.500 & pendiente a $12-95$ \\
\hline Martorell & Maxor & 3.696 & 1994 \\
\hline Mollet del Vallès & Bon Preu (Campolara) & 3.156 & 1992 \\
\hline Sant Quirze del Vallès & Alcampo & 10.764 & 1990 \\
\hline El Masnou & Caprabo & 3.304 & 1992 \\
\hline Sant Feliu de Llobregat & Caprabo & 3.015 & 1993 \\
\hline Barcelona & Alcampo & 18.176 & pendiente a $12-95$ \\
\hline Barcelona & Hipercor & 12.700 & 1986 \\
\hline Barcelona & Bon Preu (Esclat) & 5.710 & 1990 \\
\hline Barcelona & Disvahe & 2.876 & 1993 \\
\hline
\end{tabular}

GRANDES ALMACENES (no ligados a centro comercial)

\begin{tabular}{|c|c|c|c|}
\hline Sabadell & El Corte Inglés & 31.540 & 1991 \\
\hline \multirow[t]{2}{*}{ Barcelona } & El Corte Inglés (Plaza & & \\
\hline & Catalunya) & 41.541 & 1962 \\
\hline Barcelona & El Corte Inglés (Diagonal) & 39.870 & 1974 \\
\hline Barcelona & $\begin{array}{l}\text { El Corte Inglés (Portal de } \\
\text { l'Àngel) }\end{array}$ & 14.000 & 1926 \\
\hline Barcelona & $\begin{array}{l}\text { El Corte Inglés (Diagonal/ } \\
\text { F. Maciá) }\end{array}$ & 13.000 & 1967 \\
\hline
\end{tabular}

\section{CENTROS COMERCIALES}

\begin{tabular}{llcc}
\hline Badalona & Centre Comercial Montigalà & 19.563 & 1991 \\
Barberà del Vallès & Baricentro & 72.800 & 1980 \\
Barcelona & Illa Diagonal & 35.000 & 1993 \\
Barcelona & El Centre de la Vila & - & 1996 \\
Barcelona & Centre Comercial & 33.529 & 1995
\end{tabular}




\begin{tabular}{|c|c|c|c|}
\hline \multicolumn{4}{|c|}{$\begin{array}{c}\text { (Continuación) } \\
\text { HIPERMERCADOS, GRANDES ALMACENES Y CENTROS } \\
\text { COMERCIALES DE BARCELONA Y SU REGIÓN METROPOLITANA (1996) } \\
\text { HIPERMERCADOS mayores de } \mathbf{2 . 5 0 0} \mathbf{~ m 2} \text { de superficie } \\
\text { (no ligados a centro comercial) }\end{array}$} \\
\hline Municipio & Empresa & $\begin{array}{l}\text { Superficie } \\
\text { venta }\left(\mathbf{m}^{2}\right)\end{array}$ & $\begin{array}{c}\text { Año } \\
\text { inauguración }\end{array}$ \\
\hline arcelona & Maremagnum & 10.000 & 1995 \\
\hline ornellà de Llobregat & Llobregat Centre & 28.490 & 1994 \\
\hline $\begin{array}{l}\text { Tavà } \\
995\end{array}$ & Centre Comercial Barnasud & 28.700 & \\
\hline Hospitalet de Llobregat & Centre Comercial La Farga & 12.793 & 1996 \\
\hline abadell & Del Llac Center & 10.650 & 1996 \\
\hline abadell & Paddock Bulevard & 5.619 & 1996 \\
\hline
\end{tabular}

Fuente: Generalitat de Catalunya. Direcció General de Comerç Interior i Serveis.

\section{Un balance general}

Entre 1991 y 1995, con el crecimiento generalizado de la superficie comercial se dio también la polémica alrededor de las formas comerciales y de la regulación del sector dada la convivencia en el mismo de grandes y pequeños empresarios. Esta pugna se reabre ante la apertura de cada nuevo gran equipamiento por la oposición del pequeño comercio o, tal vez, mejor del comercio local, pues no todo el sector comercial que protesta puede ser considerado con propiedad como pequeño. Las reacciones oscilan entre el clásico proteccionismo y el asociacionismo o cooperación entre empresas; esta última es una reacción muy positiva pues, en el fondo, es la que permite competir ante el reto que supone la gran superficie, frenando la pérdida de clientes que son fidelizados a través de incentivos diversos, como vales de regalo o de gasolina, y sorteos de toda clase. La presión del sector comercial local no se limita en absoluto a lo estrictamente comercial, sino que activamente pasa al campo de lo político. En ese sentido la presión que se ejerce no se limita a las respectivas administraciones municipales sino que, también, se requiere la intervención de la administración autonómica, precisamente para frenar la acción conjunta del promotor de la gran superficie y de la política munici-

$$
-114-
$$


pal que suele apoyarle, con el fin de lograr la paralización de los proyectos municipales de instalar un determinado gran centro comercial. A partir de ese momento de la pugna intervienen las distintas administraciones y los tribunales de justicia ${ }^{19}$; así se intensifica el debate sobre la conveniencia o no de la expansión de las grandes superficies comerciales, la cuestión sobre los horarios de apertura al público, las formas comerciales, etc.

En este contexto se deben entender, especialmente por lo que se refiere a las poblaciones del área metropolitana de Barcelona, las vinculaciones que las administraciones municipales realizan entre una propuesta de centro comercial y su proyecto de ciudad, para el desarrollo estratégico de cada municipio. Por ello, en muchas ocasiones se puede dar la existencia de relaciones estrechas y claras entre la planificación estratégica municipal, las empresas públicas de promoción y los proyectos de grandes superficies comerciales en el ámbito local, entre otros motivos debido a la falta de otras alternativas económicas posibles a partir de la crisis de la desindustrialización. El promotor de los centros comerciales aparece así como un importante socio, que en el futuro puede colaborar en otros proyectos relacionados con las estrategias de la ciudad, lo que le convierte en agente privado con capacidad de utilización de un espacio público, que se le cede o facilita con el fin de alcanzar algunos de los objetivos de la administración municipal. Los ayuntamientos no quieren perder su parte del pastel de las grandes superficies comerciales del área metropolitana, que ha experimentado una auténtica eclosión en los últimos años; no son en absoluto despreciables las grandes cantidades de dinero y las importantes inversiones que se mueven alrededor de este tipo de pro-

${ }^{19}$ En ese sentido puede mencionarse la actuación y la toma de postura que significa el Tribunal Superior de Justicia de Cataluña anulando en la práctica la moratoria sobre grandes superficies comerciales impuesta por la Generalitat de Catalunya y anulando, asimismo, las denegaciones de permisos de apertura por parte de las Comisiones Técnicas de Equipamiento Comercial a algunas grandes superficies, todo ello ocurrido, básicamente, durante 1996. En general las sentencias cuestionan el carácter normativo de la moratoria y de los decretos de la Generalitat y, en esencia, la capacidad discrecional de ésta para denegar los permisos de apertura, precisamente por la ausencia de normativa. La consecuencia fue un desbloqueo de todos los proyectos de centros comerciales en marcha o presentados que se habían paralizado y, en la práctica, una auténtica libertad de implantación de los mismos, además se ha producido también un cierto cambio en la orientación de la política sobre el comercio del gobierno autonómico, abandonándose un tanto la tradicional protección de los intereses del pequeño comercio. Con la fusión de Pryca y Promodés, a finales de 1999, se ha vuelto nuevamente a aprobar una moratoria de un año por parte de la administración catalana. 
yectos comerciales que implican no sólo actividades puramente comerciales sino también de gestión financiera, y lo que dichas inversiones pueden suponer a largo plazo para las economías locales. Además, para los municipios la implantación de una gran superficie comercial también suele significar la ocupación del suelo industrial en desuso, previa recalificación como suelo comercial, y, en consecuencia, una mejora de sus infraestructuras urbanas y una inversión en las mismas por parte del promotor.

Resulta, en ese marco, especialmente significativo el intento de regulación estatal a través de la ley de ordenación del comercio minorista de enero de 1996. Esta ley trata de ordenar la actividad del comercio minorista, ya que en cierta medida intenta regular el proceso de introducción de las empresas internacionales de distribución en el mercado español y de dar un respiro al comercio tradicional para que pueda adaptarse al nuevo marco ${ }^{20} \mathrm{y}$ a la previsible libertad futura de horarios comerciales ${ }^{21}$, hoy por hoy muy rígidos. De hecho, en el texto legal no se habla ni de municipios ni de consumidores, lo que el legislador intenta es pura y simplemente ordenar el sector de la distribución, en el que se pueden apreciar dos sistemas diferentes y complementarios: las nuevas formas comerciales, que conllevan, también, nuevas tecnologías, y las formas tradicionales de comercio, que, a su vez, tienen una importante repercusión en el mantenimiento de la población activa.

Cabe destacar, también, una particularidad en el caso de Cataluña, en general, y del área de Barcelona, en particular. Se trata del retraso respecto al conjunto de España en la expansión de las grandes superficies comerciales. Así, en el conjunto del Estado se puede considerar el período que media entre finales de los ochenta y $1993^{22}$, la fase de implantación y expansión en el mercado de las grandes superficies comerciales y el período entre 1993 y 1996 un momento de estancamiento o recesión de dicho mercado. En el caso catalán, en cambio no se registra un paralelo de

${ }^{20}$ Paralelamente a la Ley se ha desarrollado un Plan de Modernización del pequeño comercio, de cinco años de duración; el plan incluye la inversión de unos 100.000 millones de pesetas, y prevé la concesión de créditos blandos y ayudas para integrar al sector tradicional en los nuevos modelos de distribución.

${ }^{21}$ La ley establece una limitación de horarios comerciales de apertura en festivos hasta el año 2001, además la regulación de estos horarios ha sido traspasada, también por ley, a las Comunidades Autónomas, que detentan, asimismo, la concesión de una segunda licencia de apertura.

${ }^{22}$ Fuente: Richard Ellis, Boletín sobre el Mercado de Centros Comerciales en España. Segundo semestre 1994. 
dicho comportamiento; antes al contrario, es entre 1993 y 1996 cuando se produce la expansión de las grandes superficies comerciales; como se ha visto, en la ciudad de Barcelona fue sólo a finales de 1993 cuando se inició la creación y expansión de los shopping, mientras que el cierre teórico de la primera corona metropolitana se produjo sólo a finales de 1994 e inicios de 1995. Todos estos elementos muestran que el proceso de expansión de las grandes superficies comerciales en el área de Barcelona no solo no se estanca sino que se intensifica a partir de 1996, apareciendo también, por otro lado, como en el conjunto del Estado, una orientación hacia los centros basados en el ocio y en una fuerte oferta lúdica y un tipo de galerías comerciales en las que no figuran asociadas las grandes superficies alimentarias (como es el caso del Maremagnum en Barcelona o el Paddock Bulevard y Del Llac Center en Sabadell). Es por todo ello que puede hablarse de un comportamiento diferencial y ralentizado, en relación con el resto del Estado español, del mercado de centros comerciales tanto en Barcelona como en el conjunto de Cataluña.

La situación que se ha generado en estos últimos años no es sólo cuantitativamente distinta a la anterior sino que, también, lo es cualitativamente; lo es por el contexto de transformación social y económica en el que se produce, y por el proceso que va plasmando dicha transformación en el que se integra. Estas actuaciones además se han realizado con capital y patrones internacionales, pero adaptándose a las condiciones locales. En este sentido, puede destacarse que la arquitectura ha impuesto una mayor calidad formal, en general, siguiendo la tradición barcelonesa del diseño; el ejemplo más claro es el del rascacielos yacente de l'Illa, obra de los arquitectos Rafael Moneo y Manuel Solà Morales. Además, pese a que la oferta de estas grandes superficies se basa en el uso generalizado del automóvil privado, su localización no siempre ha tenido un carácter tan periurbano como en otros países, como lo demuestra el emplazamiento de l'Illa o de Glòries, en áreas céntricas de la ciudad. Incluso las localizaciones periféricas, situadas en poblaciones de la corona metropolitana, son relativamente poco suburbanas, con las excepciones posibles de Pryca del Prat de Llobregat, de Barna Sud, en Gavà o de Montigalà ${ }^{23}$, en Badalona, ya que

${ }^{23}$ Este centro se denomina Centre Comercial Montigalà, cuenta con una superficie construida de $36.625 \mathrm{~m}^{2}$, una superficie comercial de $19.563 \mathrm{~m}^{2}$ y con unos 66 locales y $66.141 \mathrm{~m}^{2}$ de aparcamiento, cubierto y descubierto con unas 2.690 plazas; componen el complejo un centro comercial, con hiperrnercado, galería comercial y superficies medias, locales de restauración y ocio y multicines. 
se integran plenamente en el tejido urbano de las localidades en que se ubican. Finalmente, se puede añadir todavía que la periferización disminuye más ya que el acceso por transporte público y para peatones se encuentra casi siempre asegurado; en muchos casos, el planeamiento municipal ha integrado estos centros comerciales al tejido urbano, como ocurre en l'Illa y todavía más en Glòries, donde los edificios se abren claramente a las calles de los alrededores, o, en ocasiones, se funden con las infraestructuras de transportes y comunicaciones, como es el caso del desarrollo de una importante zona comercial en el ámbito del aeropuerto de Barcelona ${ }^{24}$.

Los desequilibrios, no obstante, son también muy importantes. En primer lugar, el mismo retraso en la instalación de grandes superficies comerciales ha hecho que éstas se abran en un momento de retroceso o, como mínimo, de estancamiento de los shoppings centers, a escala mundial. En segundo lugar, la rigidez de los horarios comerciales, especialmente fuerte en Cataluña, resta competitividad a estas grandes superficies, en nombre de la defensa de la pequeña y mediana empresa comercial la tradicional en Cataluña. Finalmente, y esto probablemente sea lo más importante, la multiplicación de la oferta comercial amenaza con romper la unidad del espacio social barcelonés, cuya inercia histórica había permitido hasta ahora conciliar la centralidad y el crecimiento. La multiplicación y diversificación de la oferta comercial, de ocio y de servicios puede originar así una nueva fragmentación social, a través de la cristalización de nuevas pautas del consumo, que especialicen el uso de la ciudad y no solo la residencia, para clases y grupos sociales diferentes ${ }^{25}$.

\section{Los consumidores barceloneses}

La gran incógnita es conocer, precisamente, las pautas de consumo de los barceloneses y sus cambios. Los estudios realizados hasta ahora suelen caer en la confección de tipologías, más o menos simplistas, cuando

24 Área comercial que puede aprovechar el potencial que representan los más de 10.000 .000 de pasajeros (cifra que no incluye a los pasajeros en tránsito) que recibe anualmente el aeropuerto de Barcelona.

${ }^{25}$ Es lícito preguntarse si los habitantes de la Villa Olímpica abandonan Barcelona los fines de semana, mientras sus playas se ocupan por clases populares de otros barrios.

$$
-118-
$$


no caricaturescas ${ }^{26}$, olvidando algunas variables estructurales, sobre todo de sexo y edad, que devienen más y más importantes. La estructura familiar es obviamente fundamental, pero las diferencias generacionales se establecen, esencialmente, en el entusiasmo por la innovación y rotación de los objetos de consumo y en la capacidad de compra y endeudamiento.

$\mathrm{Al}$ mismo tiempo se olvidan también las variables territoriales, muchas veces por imposición de las fuentes de estudio existentes ${ }^{27}$. Las aproximaciones parciales que se han hecho hasta ahora, muestran una cierta diferenciación local, de las pautas del consumo, por internacionales que sean los productos consumidos. En el caso del mercado español del automóvil, por ejemplo, pueden detectarse diferencias bien marcadas respecto del alemán, incluso en la compra de coches alemanes: los consumidores españoles priman el aspecto externo, sobre las prestaciones y están dispuestos a esperar mucho menos tiempo entre adquisición y recepción del vehículo, lo que obliga a los distribuidores a tener grandes stocks, con tiendas y almacenes muy grandes. En el caso de Barcelona, además, hay que tener presente que las empresas, públicas y privadas, y los visitantes, catalanes, forasteros y extranjeros, son también consumidores muy importantes, con pautas y necesidades totalmente diferentes.

Las conclusiones, por tanto, han de quedar abiertas y sólo pueden ser formuladas en forma de preguntas, como unos grandes interrogantes que guíen la investigación actual. Respecto a las pautas de los consumidores barceloneses algunas preguntas fundamentales son las siguientes: ¿cómo se comportan comercialmente los jóvenes? ¿Cómo concilian sus ideales pacifistas y ecologistas, o su indiferencia aparente, con su consumismo evidente? ¿Hasta que punto disminuye el consumo de los adul-

${ }^{26}$ Entre las aportaciones teóricas puede citarse la de Milton Santos que divide los consumidores en perfectos y pluscuamperfectos (Santos, 1987), o la de Pere López que habla de conversos, perversos y subversos. En el ámbito europeo puede citarse la bibliografía del CNRS francés (Scardigli, 1987). En 1987 ESADE financió uno de los más serios estudios de estilo de vida y hábitos de compra en el área metropolitana de Barcelona, pero solo encuestó a mujeres y las tipologías resultantes son expresivas de su escasa operatividad: reflexivos, consolidados, amargados, integrados, maduros, modestos, apáticos, vitalistas (ESADE, 1987).

${ }^{27}$ La información regular que publica el Instituto Nacional de Estadística de España sobre los presupuestos familiares, información que sería clave para conocer y trabajar, no se refiere más que a conjuntos provinciales y a grandes ciudades dentro de provincias, sin mayor precisión (Carreras, Domingo, Sauer, 1990). 
tos que temen que no tendrán pensiones públicas de vejez y han de ahorrar hoy para sobrevivir mañana? ¿Cómo actúan los marginados del mercado laboral, parados y jubilados, espectadores de las grandes ceremonias del consumo? ¿Cómo inciden los nuevos inmigrantes de etnias y culturas diferentes? ¿Cómo inciden las nuevas técnicas de venta por correspondencia, por teléfono, por televisión o por ordenador que convierten el espacio doméstico en superficie de comercio? ¿Cómo inciden los regalos y ofertas de empresas no directamente comerciales en la retracción de la compra clásica? ¿Cómo influyen la baja de calidad de las ofertas a precio único y grandes descuentos en el mantenimiento del gusto y de la distinción de los consumidores?

Respecto a las áreas de mercado, como tradicionalmente son conocidos los espacios del consumo, las preguntas se orientan en sentidos diferentes; en cuanto a la organización del espacio urbano: ¿cuál es la movilidad no obligada en el interior del espacio barcelonés? ¿Qué barceloneses no acuden ya nunca a la plaza de Cataluña? En cuanto a las áreas de influencia de Barcelona, en cambio, las cuestiones serían: ¿cuál es el papel comercial de la nueva Barcelona dentro de la Cataluña actual? ¿Cuáles son los centros de atracción de la ciudad, por grupos de edad, por niveles de renta y por lugar de residencia?

\section{Primeras conclusiones}

Pese a la importancia de estos interrogantes, y de otros muchos que irán apareciendo, algunos elementos claros se desprenden del estudio de la evolución comercial de la ciudad de Barcelona.

En primer lugar, es destacable el papel decisivo de las inercias históricas que tantas veces se han señalado y que muestran las complejas y fructíferas relaciones entre ciudad e historia. Estas inercias han podido convertir en positivo el retraso tradicional de la economía y la sociedad catalana, respecto a otras europeas y americanas, ya que, en el ámbito comercial, se ha salvado la calle, en su papel de espacio social y de relación (Jacobs, J., 1961). El eslogan que hace unos años lanzó la Cámara de Comercio de Barcelona, donde hay comercio hay vida, se ha traducido en las políticas recientes de construcción de centros comerciales a partir del espacio urbano existente, más que crear ciudades artificiales en la periferia o búnkers cerrados en los barrios. 
En segundo lugar, hay que relativizar este papel destacado de las inercias históricas, ya que la escala temporal tan pequeña del estudio que se ha realizado propicia el encuentro de continuidades; es preciso ampliar las escalas de estudio, con monografías profundas sobre áreas comerciales concretas y sobre momentos históricos determinados para poder evaluar más adecuadamente la estructura y la dinámica del comercio barcelonés. Sólo el análisis multiescalar, espacial y temporal, puede permitir la captura de esta dinámica

En cualquier caso, es necesario superar el relativo menosprecio en que se han tenido los estudios comerciales, en conjunto (Jackson, 1993). Por lo que se refiere a la metodología de análisis, los resultados de las aproximaciones hechas hasta ahora han puesto en evidencia la relativa inocuidad de los estudios tradicionales, demasiado cuantitativos, más cerca del marketing que de la reflexión problemática; estos estudios, además, generalmente utilizan datos muy poco representativos, como las licencias fiscales, o los agrupan en unidades poco significativas, como los distritos municipales. El cambio de consideración y la mejora de los resultados analíticos, permitiría, sin duda, el refuerzo de las políticas comerciales, con actuaciones decididas sobre los temas importantes, como la flexibilización horaria o el urbanismo comercial. La reconsideración de las políticas comerciales llevaría a las administraciones a mejorar las fuentes de información, cosa que influiría muy positivamente sobre el aumento de rigor de nuestros estudios, terminando así con un circulo vicioso que es preciso cambiar.

\section{BIBLIOGRAFÍA}

BAUdRIllard, J., La société de consommation, Ed. Denoël, París, 1970

BENACH, N., «Els serveis a la producció», en Carreras, C. (Dir), Geografía General dels Països Catalans, vol. 5, Ed. Enciclopèdia Catalana, Barcelona, 1994.

BENACH, N., «La promoción de nuevas actividades terciarias en la ciudad de Barcelona», Revista de Geografía, vol. XXIX, núm. 2, Publicacions de la Universitat de Barcelona, Barcelona, 1995.

Bourdieu, P., La distinction. Critique sociale du jugement, Ed. du Minuit, París, 1979.

CABRUJA, A., «Los mercados municipales: elementos de polaridad del urbanismo comercial» en AA.VV., El urbanismo comercial, Generalitat Valenciana, Paterna, 1991.

CAMPBELl, C., The Romantic Ethic and the Spirit of Modern Consumerism, Ed. Basil Blakwell, Oxford, 1987.

CARRERAS, C., «L'evolució de les activitats comercials a la ciutat deBarcelona», Revista Catalana de Geografia, núm. 15,Institut Cartogràfic de Catalunya, Barcelona, 1991. 
— «Consumo y desarrollo comercial urbano» en Sociedade e Territorio, núm. 17, Ed. Afrontamento, Porto, 1992.

- Geografia urbana de Barcelona. Espai mediterrani, temps europeu, Ed. Oikostau, Vilasar de Mar, 1993.

_ «Els serveis al consum» en Carreras, C., (Dir.) Geografia General dels Pä̈sos Catalans, vol. 5, Enciclopèdia Catalana, Barcelona, 1994.

— «Os novos espaços de consumo em Barcelona» en Finisterra, núm. 57, Centro de Estudos Geograficos, Lisboa, 1994a.

_ «Los nuevos espacios del consumo en Barcelona», Revista de Geografía, vol. XXIX, núm. 2, Publicacions de la Universitat de Barcelona, Barcelona, 1995b.

CARRERAS, C., DOMigo, J.; SAUER, C., Les àrees de concentració comercial de la ciutat de Barcelona. Barcelona ciutat de compres. Cambra Oficial de Comerç, Indústria i Navegació de Barcelona, Barcelona, 1990.

Carreras, C., Martínez, S. y Romero, J., Els eixos comercials metropolitans. Ajuntament de Barcelona, Barcelona, 1999.

CUARTAS, J. Biografía de El Corte Inglés. La historia de un gigante, Libros Límite, Barcelona, 1992.

DíEz, C. y Sobrino, F. J., «Distribución comercial en Catalunya. Evolución sectorial y actuaciones públicas», en Distribución y Consumo, diciembre 1994/enero1995.

ESADE, Estilos de vida y hábitos de compra (ejemplar policopiado), 1987.

Ferry, L., Homo Astheticus. L'invention du goût à l'âge démocrathique, Ed. B.Grasset, París, 1990 .

GISPERT, M., «Ciudad y comercio, la experiencia del Bulevard Rosa de Barcelona», en AA.VV., El urbanismo comercial, Generalitat Valenciana, Paterna, 1991.

JACKSON, P., «Towards a cultural politics of consumption», en Bird, J. et al, Mapping the futures. Local cultures, global change, Routledge, London, 1993.

JACoBS, J., The Death and Life of Great American Cities, Random House, New York, 1961.

Lipovetzky, G., L'empire de l'éphemere, París, 1986.

LiTTLE, I. D. M., «A Reformulation of Consum Behavior», en Oxford Economic Papers, New Series, vol. 1, núm. 1, 1949.

LÓPEZ, P., El centro histórico: un lugar para el conflicto, Publicacions de la Universitat de Barcelona, Barcelona, 1986.

— «1992, objectiu de tots? Ciutat-empresa i dualitat social a la Barcelona Olímpica», Revista Catalana de Geografia, núm. 15, Institut Cartogràfic de Catalunya, Barcelona, 1991.

— «Todos, mayoría y minorías en la Barcelona Olímpica. Apuntes sobre el gobierno de lo social en la ciudad-empresa», en Economía y Sociedad, núm. 9, Madrid, 1993.

_ Un verano con mil julios y otras estaciones. Barcelona: de la Reforma interior a la revolución de julio de 1909, Ed. Siglo XXI, Madrid, 1993.

McCraken, G., Culture and Consumption, Indiana University Press, 1988.

RICHARD ELLIS, Informe sobre el mercado de centros comerciales, 1995.

ROMERo, J, «Bibliografía sobre terciarización urbana, comercio y consumo», Revista de Geografía, vol. XXIX, núm. 2, Publicacions de la Universitat de Barcelona, Barcelona, 1995.

Santos, M., O espaço do cidadão, Ed. Nobel, São Paulo, 1987.

SCARDIGLI, V., L'Europe des modes de vie, Ed. du CNRS, París, 1987

TELLO, R., «Plan estratégico de Barcelona: balance y nuevo plan», Revista de Geografía, vol. XXIX, núm. 2, Publicacions de la Universitat de Barcelona, Barcelona, 1995.

- Martínez, S., «Terciarización y encarecimiento de la vivienda en Barcelona», Revista de Geografía, vol. XXIX, núm. 2, Publicacions de la Universitat de Barcelona, Barcelona, 1995. 
RESUMEN: Este articulo presenta un análisis de la evolución histórica de las formas y equipamientos comerciales de la ciudad de Barcelona y de los hábitos de consumo de sus ciudadanos. Se sientan unos primeros antecedentes a inicios del siglo xx, para detenerse en el impacto de los cambios que se suceden durante los años sesenta, de auténtica revolución burguesa tardía. A continuación se centran los cambios a partir de 1986, fecha clave para iniciar el impacto de la globalización en la economía española. Del análisis de esta evolución se realiza un balance de la situación actual, a partir del marco legal y del retraso relativo en la modernización del equipamiento comercial de Barcelona. Tras plantear algunas cuestiones relevantes acerca del comportamiento de los consumidores barceloneses se concluye una cierta capacidad de resistencia de las formas locales frente a la fuerza del modelo homogeneizante que impone la globalización.

ABSTRACT: This article presents an analysis of the historical evolution of retail forms and infrastructures in the city of Barcelona and of the cosummer patterns of its citizens. First, some antecedents are defined at the very beginnig of xxth Century, looking after to the big transformation of the sixties, the real and late Spanish bourgeois revolution. The next period corresponds to the changes started in 1986, with the integration of Spanish economy into the European Market. An evaluation of the present situation remarks the relevance of the legal constraints and of the relative delay in the development of some modern commercial forms in the City. From the study of some relevants questions on the consumers behaviour a clear conclusion appears relative to the inercia of some local trends in front of the weight of globalization. 